\title{
Computer Science: Reflection and Future
}

\author{
R K SHYAMASUNDAR \\ IIT Bombay
}

\section{Early Formative Era}

There are various evidences of calculation as an ancient activity that dates back to Babylonian days used for varieties of navigational, astronomical and other day-to-day needs. In fact, there were methods of storing like Quipu of Incas and tools for calculating like Chinese counting rods. Computing as a discipline is a recent one even though the practice of using mechanical aids for calculation can have various dates based on the perspective of the reader like Blaise Pascal in 1600s, George Boole in the 1800s or the Babylonian dates of 1800BCE. It is only in the early $20^{\text {th }}$ century a firm foundation of Computing was laid while attempting to solve the problem referred to as the Entscheidungsproblem ${ }^{1}$. Posed by David Hilbert and Wilhelm Ackermann in 1928. Alan M. Turing British mathematician established that there is no method to solve this problem through a formal definition of an abstract machine, now referred to as Turing Machine. This seminal work laid the foundation of computing. It must be mentioned that while other contemporary logicians like Alonzo Church, Emil L. Post, and A. A. Markov had proposed logical formalisms to show that the Entscheidungsproblem was not solvable and in fact, it was later shown that these formalisms turned out to be equivalent to Turing Machine's. However, it was Turing's work that gave a firm momentum to the computing field from multiple dimensions. This becomes evident from the quote due to Kurt Gödel from his Gibbs Lecture: "the greatest improvement was made possible through the precise definition of the concept of finite procedure, which plays a decisive role in these results. There are several different ways of arriving at such a definition, which, however all lead to exactly the same concept. The most satisfactory way, in my opinion, is that of reducing the concept of finite procedure to that of a machine with a finite number of parts, as has been done by the British mathematician Turing". Furthermore, Gödel accepted the earlier thesis of Church only after Turing's work. The thesis since then comes to be known as Church-Turing thesis. The thesis, which had a far-reaching impact on this field, is informally stated below:

Any algorithmic problem for which an algorithm can be found in any programming language on any computer (existing or that can be built in future) requiring unbounded amounts of resource is also solvable by a Turing Machine.

In other words, the thesis implies that the most powerful supercomputer with the most sophisticated array of programming languages is no more powerful than a PC with a simple hardware and software up to polynomial loss in efficiency. Thus, the seminal paper can be treated as the birth of Computer Science. John von Neumann engineered Turing's ideas of programs as data (the concept then referred to as Stored Program concept) to realize the first stored program computer - often referred to as von Neumann machines. These ground breaking theoretical and practical realizations essentially launched the field of Computer Science and Computer Systems that have had a great impact on science and society.

Not only did Turing invent a machine capable of computing all effectively computable functions, he

\footnotetext{
*Author for Correspondence: E-mail: shyamasundar@gmail.com

${ }^{1}$ The question posed was: Is it possible to have a method that takes a proposition in first-order logic as input which will decide in a finite number of well-defined steps, whether the proposition is true or not?

${ }^{2}$ Note that by establishing that there is no complete and consistent set of axioms for all of mathematics, Gödel shattered the dream of Bertrand Russell and A. N. Whitehead.
} 
formulated a test, which has come to be known as Turing Test for testing normal human intelligencethat initiated the area termed as Artificial Intelligence. Turing's digital forecast done in his paper "Computing Machinery and Intelligence" [Turing, 1950] gives a reflection of where the field has reached. To quote from Turing:

"I believe that in about fifty years' time it will be possible, to program computers, with a storage capacity of about $10^{9}$, to make them play the imitation game so well that an average interrogator will not have more than 70 per cent chance of making the right identification after five minutes of questioning. The original question, "Can machines think?" I believe to be too meaningless to deserve discussion. Nevertheless I believe that at the end of the century the use of words and general educated opinion will have altered so much that one will be able to speak of machines thinking without expecting to be contradicted". Quote (A).

An analysis of Alan Turing by Jim Gray (A Turing Laureate) provides a good assessment as to where the field has reached:

With the benefit of hindsight, Turing's predictions read very well. His technology forecast was astonishingly accurate, if a little pessimistic. The typical computer has the requisite capacity, and is comparably powerful. Turing estimated that the human memory is $10^{12}$ and $10^{15}$ bytes, and the high end of that estimate stands today. On the other hand, his forecast for machine intelligence was optimistic. Few people characterize the computers as intelligent. You can interview Chatter Bots on the Internet (http:// chatbotsmagazine.com/how-to-win-a-turning-testthe-loebner-prize-3ac2752250f1) and judge for yourself. I think they are still a long way from passing the Turing Test. But, there has been enormous progress in the last 50 years, and I expect that eventually a machine will indeed pass the Turing Test. To be more specific, I think it will happen within the next 50 years because I am persuaded by the argument that we are nearing parity with the storage and computational power of the mind. Now, all we have to do is understand how the mind works (!). Quote (B).

\section{Impact of Computer Science}

One of the hallmarks of Turing was that he was seeing computation everywhere: from abstract mathematics to developmental biological observations like stripes of a tiger or a zebra. He firmly established a variety of computational methods for the concrete understanding of traditional mathematical concepts specified by finitely definable approximations, such as measure or continuity. Some of his notable contributions of significance explicitly in this direction are:

- $\quad$ LU decomposition,

- Finite approximations of continuous groups,

- Computation over reals,

- Chemical basis for morphogenesis and non-linear dynamic simulation.

Around the time of this great intellectual revolution in computing as briefed above, the second World War had begun and was in full swing. Naturally, the military establishments of USA and UK had become seriously interested in automatic computations of ballistic and navigation tables as well as the cracking of ciphers. One of the most successful projects in this direction was the UK's top-secret project at Betchley Park that cracked the German Enigma cipher using several methods devised by Turing. These efforts had the good side-effect of providing a boost to the spread of computing resulting in universities offering new fields of study.

In the early stages of computer usage, the emphasis was on making computers useful. The research, education and development efforts could be broadly divided into four parts as shown in Table 2.1 .

As the use of computers reached a reasonable level of maturity, the areas of specialization like theory of computation, algorithmic analysis, data structures, numerical analysis, compiler construction, operating systems, programming methodology, artificial intelligence, software engineering, etc., evolved. It is of interest to note that the mathematical foundations pursued for the above studies, happened to be not classical analysis as is the case in science studies; it was rather logic (mathematical, computational, philosophical), universal algebra \& ordered sets, discrete structures, combinatorics. These topics could be termed "mathematics of weak structures" where 
Table 2.1: Research, Education, and Development efforts during early stages of computer usage

Characterization of computable functions/problems, intrinsic complexities of algorithms, logic of programs:

Languages for specifying algorithms and data so that they could be automatically computed in an effective manner:

Building reliable systems that can realize computations efficiently:

Artificial Intelligence:
These areas got bunched under the broad name theoretical computer science that developed the underlying mathematical foundation to support this direction of research that lead to creation of automata theory, formal languages, computability theory, algorithm analysis, logic of programs, semantics of programming languages.

These goals developed areas like programming languages, compilers, databases, etc.

The underlying goals developed areas like computer architectures, operating systems, software engineering (intellectual manageability of large programs), etc.

The efforts were to see how best the computer could mimic a human and build systems to aid human reasoning. "weak" is used in an axiomatic sense like semi-groups vs. groups, distributive lattices vs. Boolean algebras, projective vs. Eucledian geometry. These topics, perhaps due to lack of stimulating applications, have always existed as topics of peripheral interest within mathematics. The requirements of Computer Science completely changed the situation. Computer Science needed ideas from these topics and in turn stimulated the development within these topics by posing questions which would not have been posed otherwise.

Right from the days of germination of ENIAC/ EDSAC, John von Neumann had been advocating that computers would not be just a tool for aiding science but a way of doing science. With the computing reaching a stage of robustness in terms of hardware, software and user interface by early 1970s (time around which Computer Science germination happened in India - thanks to TIFR and IIT Kanpur) and the use of computers in science \& engineering gained momentum. Ken Wilson, a Nobel Laureate in Physics, promoted an idea that simulation on computers was a way to do science and scale-up discoveries and inventions. It may be noted that Wilson's breakthroughs were realized through computational models whose simulations produced radical understanding of phase changes in materials. In fact, he championed the promotion of computational science saying that grand challenges in science could be cracked through computers. He went on to call that computation has become a third leg of science. His promotions lead to formal streams under "Computational Sciences" and also government funding for building computers increased quite substantially leading to further technological advancements. It is to be noted that these initiatives lead to graduate programmes in computational sciences worldwide and the area of "High Performance Computing" took shape in academia, industry and business.

With the gearing up of science, engineering, and technological advances, areas like databases, visualization, graphics and image processing, etc., became important. The importance of human machine interface for both computer science experts and nonexperts for productivity as well as varieties of applications, including business and media, lead to the invention of personal computers at Xerox PARC. These developments galloped at high momentum and developed the area of human computer interfaces (HCI) with vast applications that made computing ubiquitous.

The success of ARPANET leading to birth of Internet, the advances in mobile technology and computing and communication coming together stimulated areas like mobile computing, security, network science, etc. With the invention of world-wide-web in the early 1990's, computing spread widely even to areas which one had not imagined and in particular e-commerce. The growth of e-commerce, use of Internet for infrastructures (online store, online payment), in numerable ubiquitous applications has lead to the new field of network and information security - that has been immensely challenging from various perspectives including national and public life.

Widespread developments along with the technological advances that brought together computing and 
communication on one platform has lead to vast set of unimaginable applications to entertainment that includes: live music, video conferencing, virtual reality, online games, $360^{\circ}$ photos, etc. These developments have been driving a revolution in Computer Science. The principle drivers of this revolution are:

- Integration of computing and communication,

- Huge volumes of digital data,

- $\quad$ The deluge of networked devices and sensors.

These developments have further triggered ways of looking at networks of people and organizations, and their integration into management, law, and policy. Concretely, the developments have given rise to a vast variety of social networks for entertainment, business, and societal governance. Needless to say, these trends have carved an entirely multi-disciplinary spectrum of challenges for integrating information systems for societal requirements.

Scaling up these computing technologies (hardware and software) with high productivity has been a huge impact on discoveries and inventions in science and engineering disciplines. In fact, we have reached to a point, wherein significant progress either in science, engineering or society is dependent on the computing power, for example, antibiotic drug discovery, study of gravitational waves or predicting next solar flares so that satellites and critical ground electronics can be safeguarded from burning. Some of the areas wherein computing has made a huge impact and expected to have disruptive impact are: smart materials, epidemiology, genomics and molecular modeling, astronomy, computational chemistry, biology, e-commerce, e-governance, health-care, robotics, earthquake engineering, disaster management, national security, public infrastructures, large-scale societal systems, etc.

The current information age is a revolution that is changing all aspects of our lives. Those individuals, institutions, and nations who recognize this change and position themselves for the future will benefit enormously. Thus, we need to position ourselves in order to drive the potential benefits to the society. The magnitude of impact made by computing to science \& society can be gauged by the highly convincing argument in the report [Tichenor, 2007], where Suzy Tichenor, Vice President, U.S. Council on Competitiveness, argues that:

\section{the country that wants to out-compete must out-compute}

In the report, it is argued that to drive the growth of innovations (hence the growth of the country) it is necessary to gain competitiveness with computational modeling and simulation. The main reasons behind that being (again quoting):

- High Performance Computing (HPC) is an innovation accelerator

- HPC shrinks "time-to-insight" and "time-tosolution" for both discovery and invention

The key takeaway argued for USA at that time, was:

enable companies, entrepreneurs, individual inventors to: innovate anywhere, with anyone, using any domain specific application running at any available High Performance Computing center.

Given that we are still to gain competitiveness in hardware and scalable computing is capital intensive - we should concentrate on building large scale systems using innovative architectures and make available to stake-holders such as companies, entrepreneurs, researchers, and individuals and give momentum in driving innovations. Some of the specific findings in terms of HPC, Big Data analytics as well as infrastructure takeaways are elaborated later.

\section{Shaping of Computing Discipline}

As computing is omnipresent, it has benefitted from the best of the talents from all disciplines. Just to mention a few in the early days of computing like, Alan Turing, John von Neumann, Claude Shannon, Alonzo Church, etc., each a towering personality in a multiple disciplines of the day. Computer Science as a discipline is not even a century old, and furthermore, due to the application strides being made by the computing, the field attracted very many people from several areas of mathematics, electrical engineering, physical sciences, economics, law, and business. Due to such a large spectrum of interests, there have been a large number of dizzying arguments about the core 
features of computing as an academic discipline. Thus, it is but natural that various views arise depending on pioneers of the field, the background training of the persons etc. Some of the common viewpoints are:

1. Computer Science is just a technological application of mathematics, electrical engineering or science.

2. Computer Science is an independent discipline with a sound body of knowledge with its own set of challenges and ultimately is the foundation of Art of Thinking.

3. Computing is primarily a technical field that aims at cost-efficient solutions.

4. Computing is an empirical science of information processes that are found everywhere.

Several early computing pioneers have argued about the nature of Computer Science keeping in view their key perspectives. An excellent discussion of these are given in Matti Tedre [Tedre, 2014]. Some of the views are briefed below:

- $\quad$ Programming is computer science (Edsger W. Dijkstra)

- Algorithmic analysis is the unifying theme (Donald E. Knuth)

- Juris Hartman in FSTTCS 1993 address discusses the nature of Computer Science as a science by analyzing it and comparing or contrasting it with other physical sciences. $\mathrm{He}$ argues that Computer Science differs from the known sciences so deeply that it has to be viewed as a new species among the sciences. This view is justified by observing that theory and experiments in Computer Science play a different role and do not follow the classic pattern in physical sciences. The change of research paradigms in Computer Science are often technology driven and simulations can play the role of experiments. Furthermore, the science and engineering aspects are deeply interwoven in Computer Science, where the distance from concepts to practical implementations is far shorter than in other disciplines.

- Herbert Simon, an economics Nobel prize winner and a Turing Laureate, called "computing" - The Sciences of the Artificial.

Over the past few decades, vast streams of insights on foundational aspects of algorithms, programming, representations of problems and languages of representation have been achieved. Feats of integrating computing and communication to build large complex, reliable systems have been realized and further, Artificial Intelligence (AI) techniques (like Deep Learning) have shown enormous potential in building real intelligent systems that mimic human intelligence (as forecasted/ envisaged by Alan Turing) like driver-less cars, robots for medicine administration or aid in disasters like earthquake, systems that can challenge and defeat human experts who play games like Chess, Go, Jeopardy!, etc. Computer modelling and simulation has made a huge impact in computational chemistry, genomics/biology analysis, smart materials, etc.

In summary, computing has been a driver in different traditions of physical sciences, engineering, mathematics and also building societal systems in the digital era. It is almost impossible to draw a line between them as the intellectual endeavors/pursuits they represent/impact are not definable. The nature of Computer Science has evolved at a rapid pace in theory, practice and applications. For instance, the relationship between Computer Science and Mathematics is nicely captured by Knuth (1994) quoted below:

Like mathematics, computer science will
be somewhat different from the other
sciences, in that it deals with man-made
laws which can be proved, instead of
natural laws which are never known
with certainty. Thus the subject will be
like each other in many ways. The
difference is in the subject matter and
approach - mathematics dealing with
more or less theorems, infinite
processes, static relationships and
computer science dealing with more or
less with algorithms, finitary
constructions and dynamic
relationships.

While the above sets the stage for evolution, the following quote from Knuth (1985) shows the limitless nature of evolution: 
I suppose the name of our discipline isn't of vital importance, since we will go on doing what we are doing no matter what it is called; after all, other disciplines like Mathematics, and Chemistry are no longer related very strongly to the etymology of their names.

The table showing the range of topics during 1968-2008 taken from [Tedre, 2014] is given in Fig. 2.1 .

There have been several views saying that Computer Science dealt with laws of nature, as well as computing is natural science [Denning, 2003] and a thorough analysis of these aspects is explored in [Tedre, 2014]. With the maturing of the discipline and the huge impact it has made, one can conclude that it has provided a way of thinking in almost all branches of science, engineering and society; the latter abstraction can be succinctly seen in the coining of the phrase "Computational Thinking" by Jeannete Wing of CMU (we shall look a bit more into this aspect in the sequel). The elucidation of such an impact along structures of science and engineering frameworks has been captured nicely by Peter Denning [Denning 2003] in Figure 2.2. One inference you can see, why the notion of "experiments" has also an important role nowadays and also fits well in the significant contributions of machine learning being played along for societal applications. In fact, these arguments and happenings are reflected in the following quote from Forsythe (1969):

\section{The question: "What can be automated" \\ is one of the most inspiring philosophical and practical questions of contemporary civilization}

While Computing has penetrated all areas as discussed already, in the following we shall take a broad look at the challenges of some of the areas that have arisen from computing for science and engineering. In particular, we focus on some of the research challenges in select areas of relevance.

Our focus of the report is to broadly highlight the role of computing science and engineering in various areas of science, engineering and societal applications. We shall discuss:
1. Computing for scaling up discoveries in science

2. Computing as a disrupter in building societal systems and the implications to the human society.

3. Broad understanding on the challenges in computing.

4. Broad view to the funders and government to organize appropriately to meet the challenges.

Keeping this in view, the report discusses the following topics:

1. A glimpse into Computer Science research challenges

2. HPC significance for science and engineering

3. Societal impact: blockchain as the protocol for trust in Internet era

4. Exploiting network computing towards such requirements

5. Big Data applications for governmental needs

6. HPC in public infrastructures

7. Re-organizing computing education for various disciplines; in this connection we append a report that was recently arrived on the sideline of a conference dedicated to Homi Bhabha.

Rest of the section provides glimpses of research challenges and developments in computing w.r.t. some select areas like: AI, MOOCs, security \& privacy.

\section{A Glimpse into Research Challenges in Computing}

In these days, with the availability of massive computations, there has been a shift of parts of decision phase of applications, that had been the forte of humans, to machines. This is referred to as $\mathrm{AI} / \mathrm{Machine}$ Learning in various glorious terms in the media. It is important that the compound annual growth rate (CAGR) of investment in such applications is more than 47 billion USD. Thus, the machines are not necessarily just number crunchers but also decision makers. For instance, driving by machines is being largely explored and indeed has 
demonstrated a reasonable success ${ }^{3}$. Thus, it is future challenges ${ }^{4}$. important to keep this in mind while addressing the

\begin{tabular}{|c|c|c|}
\hline $\begin{array}{l}\text { Computer science subjects } \\
\text { in Zadeh (1968) }\end{array}$ & $\begin{array}{l}\text { bareas of computing } \\
\text { eenning et al. (1989) }\end{array}$ & $\begin{array}{l}\text { Core technologies } \\
\text { in Denning (2008b) }\end{array}$ \\
\hline $\begin{array}{l}\text { Theory of algorithms } \\
\text { Models of computation } \\
\text { Data structures } \\
\text { Finite-state systems } \\
\text { Dynamic programming }\end{array}$ & $\begin{array}{l}\text { Algorithms } \\
\text { and data } \\
\text { structures }\end{array}$ & $\begin{array}{r}\text { Algorithms } \\
\text { Data structures }\end{array}$ \\
\hline $\begin{array}{l}\text { Programming languages } \\
\text { Automata theory } \\
\text { Formal languages and grammars } \\
\text { Programming systems }\end{array}$ & $\begin{array}{l}\text { Programming } \\
\text { languages }\end{array}$ & $\begin{array}{r}\text { Programming languages } \\
\text { Compilers }\end{array}$ \\
\hline $\begin{array}{l}\text { Switching theory } \\
\text { Computer design and organization }\end{array}$ & Architecture & $\begin{array}{r}\text { Computer architecture } \\
\text { Supercomputers } \\
\text { Parallel computation } \\
\text { Distributed computation }\end{array}$ \\
\hline Operating systems & $\begin{array}{l}\text { Operating } \\
\text { systems }\end{array}$ & $\begin{array}{r}\text { Operating systems } \\
\text { Networks } \\
\text { Real-time systems } \\
\end{array}$ \\
\hline $\begin{array}{l}\text { Discrete mathematics } \\
\text { Numerical methods } \\
\text { Mathematical programming }\end{array}$ & $\begin{array}{l}\text { Numerical and } \\
\text { symbolic } \\
\text { computation }\end{array}$ & $\begin{array}{l}\text { Computational science } \\
\text { Scientific computation }\end{array}$ \\
\hline & $\begin{array}{l}\text { ftware methodology } \\
\text { and engineering }\end{array}$ & $\begin{array}{r}\text { Software engineering } \\
\text { Data security }\end{array}$ \\
\hline Information retrieval & $\begin{array}{l}\text { Database and } \\
\text { information } \\
\text { retrieval systems }\end{array}$ & $\begin{array}{r}\text { Databases } \\
\text { Information retrieval } \\
\text { Data mining }\end{array}$ \\
\hline $\begin{array}{l}\text { Computational linguistics } \\
\text { AI and heuristic programming } \\
\text { Patter recognition and learning systems }\end{array}$ & $\begin{array}{l}\text { Artificial } \\
\text { intelligence } \\
\text { and robotics }\end{array}$ & $\begin{array}{r}\text { Artificial intelligence } \\
\text { Robots } \\
\text { Natural language processing } \\
\text { Vision }\end{array}$ \\
\hline Computer graphics & $\begin{array}{l}\text { Human- } \\
\text { computer } \\
\text { communication }\end{array}$ & $\begin{array}{r}H C I \\
\text { Graphics } \\
\text { Visualization }\end{array}$ \\
\hline $\begin{array}{l}\text { Also listed in 1968: } \\
\text { Digital devices and circuits } \\
\text { Mathematical logic } \\
\text { Information theory and coding } \\
\text { Analog and hybrid computers } \\
\text { Combinatorics and graph theory }\end{array}$ & & $\begin{array}{r}\text { New in } 2003: \\
\text { Management information systems } \\
\text { Virtual reality } \\
\text { Decision support systems } \\
\text { E-commerce } \\
\text { Workflow }\end{array}$ \\
\hline
\end{tabular}

Fig. 2.1: The Evolution of Computer Science Topics

\footnotetext{
${ }^{3}$ Two points to be kept in mind: (i) Is driving an intellectual activity?, (ii) Can machine handle ethics - for instance, when it comes to choosing an action between self protection an external person (say pedestrian) in an emergency, what will be the basis for the machine's decision? These are questions, for which there are no easy answers.

${ }^{4}$ In such a framework there has been many forecasts while predicting the future. For the scientists/technologists who invent the future, it is nevertheless important to understand correct scenarios of the real world. This is particularly important for the AI discipline, as it has had a roller coaster role. A recent article by the Turing Laureate Prof. Rodney Brooks, The seven deadly sins of AI Predictions, MIT Technology Review, 6 October 2017.
} 


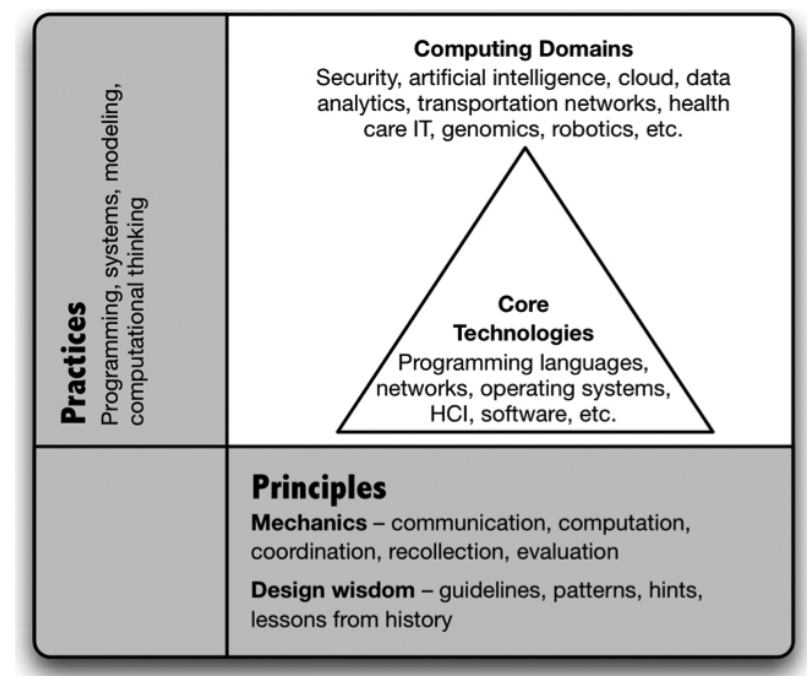

Fig. 2.2: Computing Frameworks

While there are the classical scientific research challenges like the " $\mathrm{P}=\mathrm{NP}$ " problem, in this section, we shall look at a broader perspective from the viewpoint highlighted already. We shall briefly discuss areas that are covered explicitly as separate chapters.

The address by John Hopcroft (another Turing Laureate) at the 2013 Heidelberg Laureate Forum is a good starting point, as it articulates the shift in focus of Computer Science becoming more applicationoriented in the years to come. He argues that the following topics would be some of computational challenges for the decade:

- $\quad$ Tracking evolution of communities in social networks

- Extracting information from unstructured data sources

- $\quad$ Processing massive data sets and streams

- $\quad$ Extracting signals from noise

- $\quad$ Tracking the flow of ideas in scientific literature

- Dealing with high dimensional data and dimension reduction

It is apparent from the topics that challenges relate to inferences on data and in a sense correspond to the Big Data analytics or machine learning. Natural outcome of the challenges is the need of building a theory to support new directions. This naturally calls for a re-look into computer science education. As extrapolated by John Hopcroft, Computer Science would need to include topics like large complex graphs, spectral analysis, high dimensions and dimension reduction, clustering, collaborative filtering, learning theory, sparse vectors, signal processing, etc.

From the above perspective, an immediate broad take-away is:

"Introduce computing paradigms and methodologies in schools and colleges, and revisit curricula of science, engineering and humanities (UG and PG) to provide the needed ICT paradigms".

Keeping in view, that various challenges in areas like HPC, SDNs, Big Data, smart-grid, etc., are covered in other chapters. In this section, we shall provide glimpse of some of the challenges in areas like AI, MOOCs, and security \& privacy that could have a disruptive impact on science and society.

\section{Artificial Intelligence}

"... if a machine is expected to be infallible, it cannot also be intelligent"

A. M. Turing, London Mathematical Society Address, 20 Feb 1947.

"Artificial Intelligence" was coined by Alan M. Turing through the formulation of a test which has come to be known as Turing Test for testing normal human intelligence. The Turing Test is an imitation game, played by three people. In this game, a man and a woman are in one room, and a judge is in the other. The three cannot see one another, so they communicate via e-mail (letters/notes). The judge questions them for 5 minutes, trying to discover which of the two is the man and which is the woman. This would be very easy, except that the man lies and pretends to be a woman. The woman tries to help the judge. If the man is a really good impersonator, he can fool the judge $50 \%$ of the time. But, it seems that in practice, the judge is right about $70 \%$ of the time. Now, the Turing Test replaces the man with a computer pretending to be a human. If it can fool the judge $30 \%$ of the time, it passes the Turing Test. The rationale of the test may be seen in Turing's own words in his BBC interview:

\section{The idea of the test is that the machine}



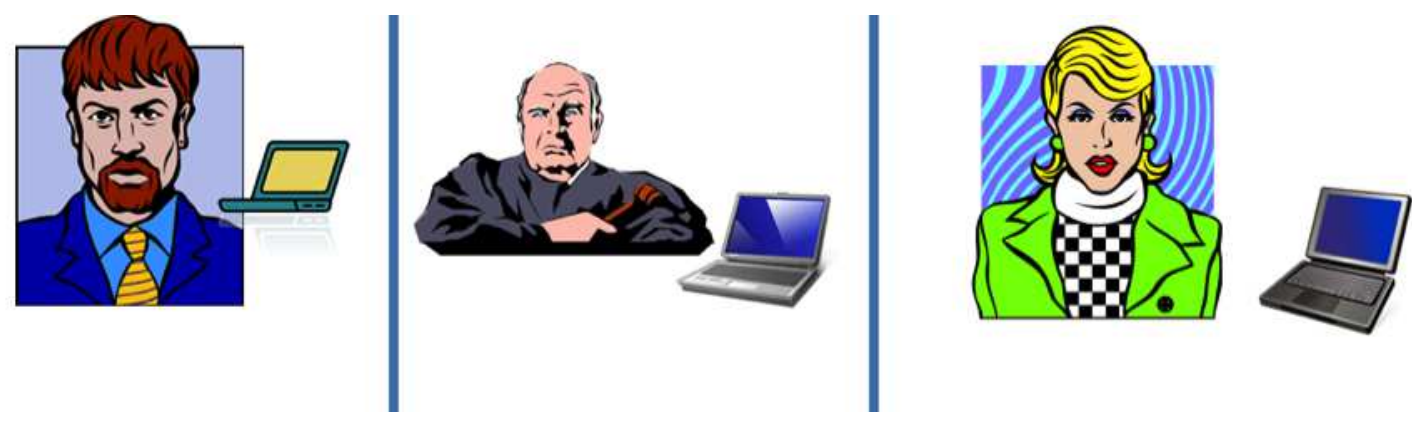

Fig. 2.3: Turing Test for Normal Human Intelligence

has to pretend to be a man, by answering questions put to it, and it will only pass if the pretence is reasonably convincing ... We had better suppose that each jury has to judge quite a number of times, and that sometimes they really are doing with a man and not a machine. That will prevent them from saying 'It must be a machine' every time without proper consideration.

The underlying arguments against the test can again be seen in his own words:

The game may be criticized on the ground that the odds are weighted too heavily against the machine. If the man were to try and pretend to be the machine he would clearly make a very poor showing. He would be given away at once by slowness and inaccuracy in arithmetic. May not machines carry out something which ought to be described as thinking but which is very different from what a man does? This objection is a very strong one, but at least we can say that if nevertheless, a machine can be constructed to play the imitation game satisfactorily, we need not be troubled by this objection.

Even though it is not formally defined, it is a practical test applied to an existing entity that is "running". It consists of a conversation over a period of time between the tester and the entity being tested. This demands an ability to learn and adapt the contents and the structure of the sayings of the tester. Note that the testing becomes harder the longer it goes on.
The point of the test is that if some entity passes it, it is hard to deny that it is intelligent and hence throws up the possibility of judging artificial entity to be intelligent. The basis for this is based on Turing's view that "thinking is singularly and critically indicated by verbal behavior indistinguishable from that of people as determined by a blinded experiment." In summary, Turing Test shares important properties with interactive proofs such as exponentially rare false positives, non-composability, non-transferability, etc. Turing's seminal contribution was in enabling blinded controls. While the Test can provide an interactive proof of intelligence, it is not particularly useful as a research goal itself. While several Internet sites offer Turing Test chatterbots, none pass and still stands as a long-term challenge. The movement of AI becomes clear if we re-look at the Quotes (A)-(B) due to Alan Turing and Jim Gray respectively.

Implicit in the Turing Test, are two subchallenges that in themselves are quite daunting:

- Read and understand as well as a human,

- Think and write as well as a human.

Both of these appear to be as difficult as the Turing Test itself. Due to advances in computing technology, there has been tremendous progress in speech recognition ${ }^{5}$ understanding, speech synthesizers, limited language translation, visual recognition, visual rendering, etc. While one may say the conceptual progress in these areas is limited, it is still a boon to the handicapped and in certain industrial settings. There is no doubt that these prosthetics have helped

\footnotetext{
${ }^{5}$ It is of interest to look at the recent claim of Microsoft (https:/ /goo.gl/CD9wHD) that claims its speech recognition has attained human parity.
} 
and will help a much wider audience and shall revolutionize the interface between computers and people. In fact, it has made a tremendous progress in the above (Google Glass is an example) as well as in smell measurements and odor reproduction (cf. Harel [R. Haddad and Sobel, 2008, Harel, 2016]). When computers can see and hear, it will break communication barriers. It should be much easier and less intrusive to communicate with them. In a sense, it will allow one to see better, hear better, and remember better. In the past couple of years, we have been seeing successes in these aspects.

In the following, we briefly highlight several of the rapid strides in some of these areas through the eyes of Artificial Intelligence.

\section{Whither Artificial Intelligence?}

While there are several examples wherein computers have assisted in arriving at proofs of several open problems in mathematics including the four color conjecture, it is the defeat the human experts in games like Chess, Go or Jeopardy! by computers that have ignited Artificial Intelligence in the eyes of public and business [Harold 2016].

It began with IBM's Deep Blue computer beating Gary Kasparov, the then reigning world chess champion. It is of interest to note that Kasparov was at a significant disadvantage during the match as the designers of Deep Blue had the opportunity to tweak Deep Blue's programming between matches to adapt to Kasparov's style and strategy. Further, they had access to full history of his previous public matches. However, Kasparov has no similar record of the machine performance as it was being modified

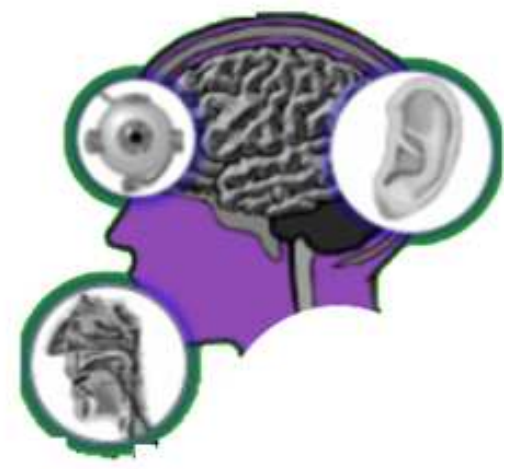

Fig. 2.4: Three Prosthetic Challenges: Vision, Hearing, and Speech between matches. Further more, Kasparov and other chess masters blamed the defeat on a single move made by the IBM machine. In that move, the computer made a sacrifice that seemed to hint at its long-term strategy. Kasparov and many others thought the move was too sophisticated for a computer, suggesting there had been some sort of human intervention during the game. In respect of that move grand-master Yasser Seirawan told WIRED in 2001, "It was an incredibly refined move, of defending while ahead to cut out any hint of counter-moves," and further he added "it sent Gary into a tizzy". Later, one of the designers of Deep Blue admitted that it was a bug that made Deep Blue make a random move.

While it established that a machine could play Chess like a champion, several people expressed whether the same strategy would work for games like Go as the choices at each of the points were horrendously large. Certainly the achievement was laudable and significant, philosophically there were apprehensions whether it really solved the problem of intelligent chess programs that had been the goal right from the early days of CS/AI. In this connection, we quote a remark of John McCarthy — a pioneer of Artificial Intelligence.

Alexander Kronrod, a Russian AI researcher, said Chess is Drosophilae of AI. He was making an analogy with geneticists' use of that fruit fly to study inheritance. Playing chess requires certain intellectual mechanisms and not others. Chess programs now play at grand-master level, but they do it with limited intellectual mechanisms compared to those used by a human chess player, substituting large amounts of computation for understanding. Once we understand these mechanisms better, we can build human-level chess programs that do far less computation than do present programs...

Interestingly, newspaper interview of David Harel, another distinguished scientist, with the title Why is it easier to beat Kasparov than to beat Turing? ${ }^{6}$ in response to the news "Deep Blue Beats

${ }^{6}$ D. Harel, "Why is it easier to beat Kasparov than to beat Turing?" (in Hebrew), in Z. Yannai, ed., The Infinite Search: Conversations with Scientists, Am Oved Publishers, Tel Aviv, 2000, pp. 48-56 
Gary Kasparov" speaks of the inventiveness of Alan Turing!

While the achievements of beating the chess champion by a computer program (or infrastructure) were not the "end goals" themselves, it brought out the power of massive computer infrastructure and the learning/feedback/inference from behavior patterns. Needless to say in this new millennium this has made big impact on science and society.

Moving from Chess, let us look at the next achievement again by IBM that built a "cognitive" system, Watson, that debuted in a televised Jeopardy!, challenged and defeated the show's two greatest champions. The challenging goals for Watson were to answer varieties of questions such as puns, synonyms and homonyms, slang, and jargon posed in possible subtle uses of natural language. As it was not to be connected to the Internet for the match, there was a need for it to amass knowledge through years of persistent interaction and learning from a large set of unstructured knowledge. Using machine learning, statistical analysis or natural language processing, it was required to understand the clues in the questions, compare possible answers, by ranking its confidence in their accuracy, and respond - all in about three seconds. Indeed, a challenging feat. The Watson indeed conquered Jeopardy! in 2011.

As highlighted already, conquering Jeopardy! was not the goal. It was the start to initiate cognitive applications that would be welcome in the society. IBM is using the realized technology to build newer generations of Watson so that it can be effectively used in oncology diagnosis by health-care professionals, and in varieties of customer services as a support representative. Currently, it is spread across the cloud with different "avatars" that can serve simultaneously a spectrum of customers across the world accessing it via phones, desktops, or data servers. As the AI improves with the feedback and hence with the usage, one should see Watson becoming smarter; anything it learns in one instance can be immediately transferred to the others. Thus Watson is now an aggregation of diverse software engines - its logic-deduction engine and its languageparsing engine might operate on different code, on different chips, in different locations - all cleverly integrated into a unified stream of intelligence. IBM provides access to Watson's intelligence to partners, helping them develop user-friendly interfaces for subscribing doctors and hospitals. Alan Greene, chief medical officer of Scanadu - a start-up that is building a diagnostic device inspired by the Star Trek medical tricorder and powered by a cloud AI, says:

\section{I believe something like Watson will soon be the world's best diagnostician - whether machine or human... At the rate AI technology is improving, a kid born today will rarely need to see a doctor to get a diagnosis by the time they are an adult.}

One other important research that is pursued at IBM that has been under the broad umbrella of "Cognitive Computing" is the brain inspired computers [Preissel et al., 2012] lead by Dharmendra Modha. The multi-disciplinary, multi-institutional effort lead by Dharamendra Modha, has lead to architectures, technology, and ecosystems that break paths with the prevailing von Neumann architecture and constitutes a foundation for energy-efficient, scalable neuromorphic systems.

The next milestone perhaps has been the mastering of the game Go with Deep Learning technology. Deep Learning techniques allow a computer system to connect the dots from different areas of knowledge akin to how the brain works to arrive at the best possible. The game of Go has long been viewed as the most challenging of classical games for AI, owing to its enormous search space and difficulty of evaluating board positions and moves. Recently, in a full-sized game of Go, a (human) professional Go player was defeated by a neural network; the point to be noted is that the neural network was trained by a novel combination of supervised-learning from human Go experts \& reinforcement-learning from ALPHAGO [Silver et $a l ., 2016]$ self-play games. It is a feat previously estimated to be at least a decade away!

As predicted by Alan Turing, AI has reached to a significant level of language, image, and speech understanding systems (even smell measurements) that have shown enormous applications in the society - truly reflecting actions by a human of a good intellect. This has shown enormous potential for societal applications. To get a view of the status of 
"Machine Learning" (in a true sense Artificial Intelligence) that has taken deep roots in science and engineering, a brief discussion is given below.

Geoff Hinton [LeCun et al., 2015] highlights the underpinnings of the successes in Natural Language Processing (language translation), Image Classification, etc. In the real world, there is a range of learning tasks starting at a typical statistical analysis or inference to Artificial Intelligence. For instance, typically, statistical analysis is characterized by:

- Low-dimensional data (e.g., less than 100 dimensions).

- Lots of noise in the data.

- There is not much structure in the data, and a fairly simple model can represent what structure there is.

- $\quad$ The main problem in the context is distinguishing true structure from noise.

On the other end of the spectrum, the task typically has the following characteristics:

- High-dimensional data (e.g., more than 100 dimensions).

- The noise is not sufficient to obscure the structure in the data if we process it right.

- There is a huge amount of structure in the data, but the structure is too complicated to be represented by a simple model.

- The main problem is figuring out a way to represent the complicated structure so that it can be learned. With the remarkable capability of these Deep Learning neural networks, one has made remarkable advances in speech and image recognition, natural language translation, driver-less cars, etc. What has really stunned AI experts, has been the magnitude of improvement in image recognition. Google has indeed become a center for Deep Learning and related AI talent.

While the above discussion shows what the pioneers of Computer Science were looking for in the building of Chess playing machines, one of the remarkable inferences that can be drawn from the various successes of computing machines beating human champions are the demonstrations of:

- Excellent engineering and experimentation with a deep knowledge of the domain,

- Capability of building a massive computing infrastructure to realize the goal.

While the prophecies of Alan Turing have come true, extending Deep Learning into applications beyond speech and image recognition will require more conceptual and software breakthroughs, not to mention many more advances in processing power (reflect on the computing power of Google).

While the achievements in speech and image understanding, natural language translation have stunned the scientists and public alike, another exciting area of work has been the interactive learning through computing related to evolution of life, pioneered by Leslie Valiant - another pioneer of Computing. Valiant [Valiant, 2013]; proposes the notion of ecorithms, which unlike most algorithms, can be run in environments unknown to the designer, and learn by interacting with the environment how to act effectively in it. Thus, after sufficient interaction they will have expertise not provided by the designer, but extracted from the environment. The model of learning they follow, known as the probably approximately correct model [Valiant, 2013], provides a quantitative framework in which designers can evaluate the expertise achieved and the cost of achieving it. Valiant argues that these ecorithms are not just a feature of computers but imposition of such learning mechanisms, determines the character of life on Earth. The course of evolution is shaped entirely by organisms interacting with and adapting to their environments. This biological inheritance, as well as further learning from the environment after conception and birth, have a determining influence on the course of an individual's life. Thus, such a line of study shall lead to a unified study of the mechanisms of evolution, learning, and intelligence using the methods of Computer Science.

\section{Takeaways}

1. There is a need to address the basic conceptual challenges in AI by core researchers.

2. Supporting the use of AI for varieties of societal benefits (hence, could use a PPP model); a 
recent report on "The First Report of the 100 Year Study on Artificial Intelligence (AI100)" has been released very recently [A1100, 2017].

3. Machine Learning and Deep Learning has been offered as a standard package on varieties of systems. In fact, India should push to bring a viable HPC-Deep Learning/Machine Learning for a varieties of applications like cyber security, DNA analysis, translation, service delivery for illiterate/layman, and also science and engineering applications that require from skeletal to deep computing. India is one of the poor investors in technology, research \& education even when considered among the G20 countries. A serious push should be made for deriving the benefits through innovations and discoveries from such an investment.

\section{Massively Online Open Courses (MOOCs)}

MOOCs is the result of the hypothesis that Internet has the potential of becoming the touchstone of education, disruptively changing the face of education. MOOCs offer free, high quality, university course content to anyone with an Internet access. These courses have been drawing tens of thousands of students to a single section. As it requires only a computer and Internet access to enroll, MOOCs can be used for continuing education courses and creditbearing under-graduate courses, leading to degree programs and even graduate education. Such a technology is indeed attractive from two perspectives:

- $\quad$ Huge scaling up of education at all levels leading to huge economic advantages in particular for developed countries,

- It is naturally an attractive option for countries like India that has a huge population residing in rural areas with an acute shortage of qualified faculty/instructors (this is true even for urban elite centers).

MOOCs hit the headlines through an online course on Artificial Intelligence offered from Stanford, instructed by Peter Norvig and Sebastian Thrun, with a worldwide enrollment of 165000 . New MOOC centers like Khan Academy, UDACITY (www.udacity.com), Coursera (www.coursera.org) have the success rate of completion at just $8 \%$. While these "universities without walls" have the potential to transform literacy, awareness of public education, and formal education, there are significant unresolved issues relating to their educational quality and financial sustainability.

\section{Challenges in MOOCs}

- Evaluation:

- Frequency - frequent appraisals are needed to make sure that the students have understood the material presented.

- Presentations augmented with laboratories, plausibly virtual laboratories:

* Application of concepts learnt from the lecture presentations in a virtual laboratory environment.

* An effort in India called Colama (www.coriolis.com) has been able to provide virtual laboratories.

* Raspberry programming systems have become widely used to support practical experiments on theories learned online.

* One of the interesting experiment has been a course on Design and Analysis of Cyber Physical Systems offered at UC Berkeley (http://leeseshia.org/). A major characteristic of the course is on the interplay of practical design with formal models of systems, including both software components and physical dynamics. Students applied concepts learned in lectures on programming a robotic controller in a specially-designed virtual laboratory environment with built-in automatic grading and feedback mechanisms.

- The factors discussed above play a vital role in deciding how students can be given credit and graded.

- A comparison of effectiveness of MOOCs in comparison with that of traditional structure:

- Devise ways to compare performance of students' learning via MOOCs as against those taking traditional courses? 
- While MOOCs would serve the paradigm of "Life-Long Learning", as it stands the traditional or the universities with traditional teachers shall remain main contributor, at least, for higher education.

- Noting that faculty-student interaction plays a vital role in traditional learning, it is not clear whether that affects at different levels of MOOC learning.

- In the MOOC world, detection of cheating by students (and thereby their assessments) is quite a challenge.

\section{Status of MOOCs}

With no tuition fees required, the convenience of online learning, and access to world-class faculty, MOOCs have the potential to draw vast numbers of students away from traditional bricks-and-mortar universities. The sheer economics of MOOCs attracts a large number of students, and several organizations are investing to build viable systems to cater to the requirements. While current MOOC offerings are targeted to the undergraduate market, there shall be a limited number of professional-, graduate-, and even doctoral-level MOOCs. While even in India, one sees signs of reluctance and disappointment on behalf of students, instructors, and universities, there is a growing feeling of being useful for skill development and training. Certainly, as we proceed, all universities shall use MOOCs in some way or the other - to provide prerequisites or some interdisciplinary training requirements.

A significant migration of students to MOOCs would threaten the viability of some MOOCs and also threaten to change the role of faculty, student, and teaching assistants and the nature of the university. For example, one quality metric for traditional universities is the average number of students per class, with a lower ratio considered desirable. Automated course delivery and grading allows for immense up-scaling of course enrollments. Does the growth of MOOCs mean we will need fewer professors but more teaching assistants? We believe that there may be pressures on traditional universities to scale course sizes by adopting partial MOOC attributes (e.g., more automated grading) but still preserving a high level of instructor-student interaction.

\section{Takeaway}

MOOCs have the potential to transform the higher educational landscape, but it is too soon to tell how significant this impact will be. MOOCs will likely play a future role predominately in continuing education, course prerequisites, and, on a limited basis, creditbearing courses. It is unlikely, but possible, that complete credit-bearing courses from accredited universities will be available through MOOCs before 2022.

\section{Security \& Privacy}

We never are definitely right We can only be sure we are wrong Richard Feynman: Lectures on the character of Physical Law.

Dependence on inter-networked computing systems in this ubiquitous world has been growing in leaps and bounds. The dependence on such systems is true for all entities: be it business, corporation, government, military, infrastructure (communication, energy, healthcare, transportation, elections, finance, et al.,) not even the common man is excluded. The most interesting observation is: none of the systems of such networked systems are indeed trustworthy by themselves. More than that, all of them are under continuous active and deliberate attack from attackers ranging from a single individual to a nation-state (government). The loss of property, business, or life due to the attacks in cyber space is enormous and ever-growing.

The over dependence of the communities and the society at large makes it mandatory to secure these inter-networked systems and defend them from attacks. A secure system must defend against all possible attacks - including those unknown that could come out in future. As defenders, having limited resources, they develop defenses only for attacks they know about. The result is new kinds of attacks are then likely to succeed. While the costs of securing these IT systems have grown overwhelmingly over the years, the direct/indirect losses, due to attacks have grown in a significant way. Thus, our adopted engineering practices as well as defenses have not succeeded; in fact, they have failed. Thus, the challenge is to provide holistic approach to attack/ fraud prevention to realize a safe inter-networked world. 
As highlighted in [Schnedier and Savage, 2009], the core of the problem of failure is inherent in the nature of security itself. Security is not a commodity like computer and communications hardware and software. It cannot be scaled simply by doing more. Security is holistic - a property of a system and not just of its components. Even a small change to a system or a threat model can have catastrophic consequences to its security. The familiar and predictable technology curves by which computer processing performance, storage, and communication, scale over the time, cannot be applied to security. Security does not follow such a model. In fact, security is characterized by following asymmetries:

- Defenders are reactive, attackers are proactive.

- Defenders must defend all places at all times, against all possible attacks (including those not known to the defender); and

- $\quad$ Attackers need to only find one vulnerability, and they have the luxury of inventing and testing new attacks in private, as well as, selecting the place and time of attack at their convenience.

- New defenses are expensive, new attacks are cheaper.

- Defenders have significant investments in their approaches and business models, while attackers have minimal sunk costs and thus can be quite agile.

- Defense cannot be measured, but attacks can be.

- $\quad$ Since we cannot currently measure how a given security technology or approach reduces risk from attack, there are few strong competitive pressures to improve these technical qualities. So vendors frequently compete on the basis of ancillary factors (e.g., speed, integration, brand development, etc.)

- Attackers can directly measure their return-oninvestment and are strongly incentivized to improve their offerings.

Research on cyber security can be summed up by: Security never settles on a claim. Every security claim has a lifetime. This fact provides a basis for setting an agenda for cyber security research. To quote Fred Schneider [Schneider, 2012]:

Medicine is an appropriate analogy, since despite enormous strides in medical research, new threats continually emerge and old defenses (e.g., antibiotic) are seen to lose their effectiveness. As the nation pursues opportunities for sustainability, healthcare, and commerce, there will be ongoing needs for cyber security research or else the trustworthiness of these systems will erode as threats evolve.

\section{Takeaways}

A broad take-away from this broad perspective is summarized below. Further, cyber security is not purely a technology problem, nor it is purely a policy (economic or regulatory) problem. The basis of security is building trustworthy systems, which requires combining technology and policy. In fact, it is for this very reason there is a need of articulating a Cyber security Doctrine that would specify the goal and means of realizing cyber security in India. A synergy in the understanding of technology, law, economics and investment policies is needed to set up a clear Cyber security Research agenda that has appropriate research, development, assessment, measurement, and deployment components.

\section{A Broad Landscape of Cyber security Issues}

There has been a wide range of significant contributions by the scientific community across academia, industry, business, government, etc., to realize trustworthiness in terms of varieties of parameters like authentication, access-control, availability, confidentiality, privacy, etc. Towards this, there has been works in areas like: (1) cryptography and PKI (public-key cryptography), (2) analysis of code for vulnerabilities, (3) malware/virus patterns via data mining, machine learning, (4) hardware/ software firewalls, intrusion detection systems, etc.

In the following, we shall highlight a few of the general class of problems that need to be addressed to overcome the evolution, maturation and diversification of threats, attacks and fraud strategies to realize a secure cyber space. 
1. Static Defense Mechanisms: Most of our approaches are reactive that have severe limitations. Instead, it would be a challenge to transform systems into safely protected systems.

2. Governed by slow and deliberative processes: security patch deployment, testing, episodic penetration exercises, and human-in-the-loop monitoring of security events.

3. Adversaries do greatly benefit from the above situation.

4. Attackers may continuously and systematically probe targeted networks with the confidence that those networks will change slowly if at all.

5. Adversaries have the time to engineer reliable exploits and pre-plan their attacks. And, once an attack succeeds, adversaries persist for long times inside compromised networks and hosts.

6. Hosts, networks, software, and services do not reconfigure, adapt, or regenerate except in deterministic ways to support maintenance and uptime requirements:

(a) Malware Trends: Infection mechanisms (malware) are on the rise either due to the vulnerabilities in the environment or due to creation of new infection mechanisms. It is quite evident that malware is still the most dangerous threat to enterprises, governments, defense, financial institutions, and the end-users. While catastrophes caused by it have lead to better preventive technologies, cyber theft has stayed ahead of these technologies due to the undecidability of the general problem of prevention, by discovering new loopholes in the underlying hardware/software systems, and arriving at new mechanisms to evade the existing detection methods. This becomes clear if we look at the general trend of malware in 2014 (http:// www.slideshare.net/ibmsecurity/the-topmost-dangerous-malware-trends-for2014):

(b) The source code for a crime kit, CarberpTrojan (widely used by the underworld) became an open, leading platform to develop similar crop of new Trojans and crime-ware kits. The new invariants would have characteristics that can be quite new and makes it very difficult to be detected by the prevalent virus detectors. In other words, malware is being commoditized.

(c) Mobile SMS forwarding malware are becoming prevalent. Thus, SMS - the basic 2FA authentication - that is widely used in financial sector gets completely compromised.

(d) Malware attacks the victim's device itself rather than remote devices.

(e) Evasion of malware analysis developed by researchers.

(f) Security of infrastructures: Due to technological advances, it has been a common practice for quite some time to use embedded computers for monitoring and control of physical processes/plants. These are essentially networked, computer-based systems consisting of application-specific control-processing systems, actuators, sensors, etc., that are used to digitally control physical systems (often in a federated manner) within a defined geographical location such as power plants, chemical plants, etc. Different terminologies like distributed control systems (DCS), cyber physical systems (CPS), supervisory control and data acquisition systems (SCADA), etc., are used for denoting similar usages. SCADA have evolved from special purpose closed system of the early era to a network of components-off-the-shelf systems consisting of computers and communication components using TCP/IP. While it has greatly enhanced the flexibility and usability, it has also exposed itself at several vulnerable points.

7. Technology has further made it possible to federate/integrate heterogeneous (built by different manufacturers) systems. While such 
capabilities have provided the needed flexibility and usability, it has also created challenges for system designers/integrator, not only from the correctness point of view but also from the point of view of security and protection of the underlying physical plants. With the arrival of complex malware (APT - advanced persistent threat), it has become very challenging to secure network and information systems from intruders and protect the systems from attackers. Recently, complex malware like Stuxnet, Flame, etc., have specifically targeted SCADA of public infrastructures like power grids/plants, and thus, bringing to the forefront the challenges in securing and protecting SCADA. The above mentioned malware are horrendously complex and hence, need a wholesome approach for detection and protection.

(a) Internet of Things (IoT): IoT has emerged as a global Internet-based technical architecture that has deeply facilitated the exchange of goods and services in global supply chain networks. If one uses the broad definition of IoT, it encompasses home automation, industrial SCADA, connected vehicles, smart meters, implantable medical devices, etc., and in a sense, the backbone of smart cities. This is quite a large coverage [Mirai DDoS attack that used IoT devices to produce DDoS traffic of $620 \mathrm{Gbps}$ ] and hence, security and privacy assurance in IoT is quite challenging given that it covers not only domains of IT but also other specific application domains.

(b) Privacy issues: Rapid advances in digital technologies and communication have lead to modern systems such as varieties of social media networks like Facebook, Twitter, etc., mobile computing platforms, and wearable devices (Google Glass, Oculus Rift), which in turn have brought new benefits to almost all aspects of our lives. For example, personalized content or service recommendations like Netflix, AdSense, NewsFeed that are dependent on collection of users' data (inferred preferences) through various direct/indirect channels, often with users' consent. Users get relevant content during their search, relevant match of service while searching on the web. It saves users' time and money. Such an immensely attractive benefit is also plagued by both conventional and emerging threats to security and privacy. In the context of web, for example, a large amount of personal information about individuals that is being collected, used, and shared across organizations, is a threat to privacy thus undermining trust, with potential to surveillance by foreign players - at times influencing democratic elections. This is a serious issue in regulated sectors like health, finance, insurance, etc. In these cases, the organizations need to assure the compliance of privacy even when the data traverses from one social/web media to another one that may have distinct privacy policies.

(c) Usable Privacy: The de facto standard to address expectations of "notice and choice" on the Web is natural language. The users usually agree to the policies even before reading the policies as these are neither easy to understand nor the user finds it relevant. Initiatives to overcome this problem with machine-readable privacy policies or other solutions that require website operators to adhere to more stringent requirements have run into obstacles, with website operators showing reluctance to commit to anything more than what they currently do. One of the challenges is to combine machine learning, natural language processing and crowdsourcing to semi-automatically annotate privacy policies in order to provide users with succinct privacy notices like the one used for energy efficiency of electric appliances - 5-star rating.

\section{Broad Challenges for Cyber Security}

To realize a firm cyber security is to provide a holistic approach to fraud prevention. This requires disruptive approaches to handle infections and cyber crime. In the following, we briefly outline some general approaches to address the issues discussed in the 
previous section on a similar structure:

(a) Dynamic Cyber Defense: The basic approach is to move to proactive defense. Two of the widely used strategies are "moving targets" or "cyber kill chain" (cf. [Okhravi et al., 2013] for details). In the former, the idea is to protect various entities like applications, OS, machine, network, session, traffic or data through various techniques including coding. In the latter that is cyber kill chain, various phases like reconnaissance, access, attack launch or persistence are identified and moved. It must be noted that the above mentioned strategies have several limitations [Okhravi et al., 2013] that need to be addressed effectively. One example, covert channel's prevention needs dynamic strategies as such channels are almost unbounded. The recent story of the cloud hosting giant Akamai Technologies that dumped journalist Brian Krebs from its servers after his website came under a "record" cyber attack [Mirai botnet; DDoS attack] is an eye opener (http://krebsonsecurity.com/2016/09/ krebsonsecurity-hit-with-record-ddos/)

(b) Guaranteed Leak-Free Information: Flow in Multi Level Security (MLS) Systems: In MLS, there is a need for correct integration of Mandatory Access Control (MAC) and Discretionary Access Control (DAC) and assure the flow of information as per the hierarchy of trusted and untrusted objects/subjects. Typical systems wherein this is important are that of operating systems (which is vital for almost any application) and cloud service brokers. In other words, the challenge is to build systems wherein a trusted party interact with an untrusted party without getting infected.

(c) Malware, Fraud, and Crime Detection: Big Data analytics has emerged as a key player in the security arena with several applications in areas like homeland security and cyber security. Big Data applications are being deployed to identify the most critical and actionable items of intelligence in near real-time. It is now considered a crucial element in detecting and deterring emerging threats. Big Data analytics in this field includes proactive data mining, data fusion, and predictive analytics techniques that are applied to all available data to gain useful insights.

(d) Secure infrastructures: In these scenarios, apart from the classical IT security, there is a need to look at other plausible new attacks considering the domain of the physical systems in conjunction with the capabilities of the embedded computers, and arrive at methods of protection and risk evaluation.

(e) Security of IoT: IoT architectures resilient to attacks, data authentication, access control and client privacy are the need of the time.

(f) Privacy: In the two reports published in November 2014 [Alkhatib et al., 2014, Mandiant, 2014], analysts estimated that the IoT (Internet of Things) will represent 30 billion connected things by 2020, growing from 9.9 billion in 2013 . These connected things are largely driven by intelligent systems (including organizations incorporating BYOD policy — Bring Your Own Device) - all collecting and transmitting data. This connectivity is changing the way we live and creating new questions about personal privacy, marketing and Internet security, as the things are manufactured and sold to consumers. A couple of challenges are:

(a) To have controlled privacy over web and social media there is a need to arrive at distinct privacy policies whose compliances can be verified either statically or dynamically.

(b) With the growth of Big Data and Analytics, there is a need to arrive at a trade off between security and privacy among varieties of stake-holders that include people, businesses, government, and malevolent actors so that each of the groups decide about releasing certain information to government, merchants, and even other citizens and to consider the consequences of every activity in which they engage.

(g) SNS as tool for information weaponization: Social Network System, have shown potential to rapidly disseminate unverified news information by nodes in the network. This has 
serious potential to swing the public opinion in either directions.

(h) Cyber security Doctrine: Succession of doctrines advocated in the past for enhancing cyber security like prevention, risk management, and deterrence through accountability have not proved effective. There is a need to learn from failed doctrines and study the possibility of viewing cyber security as a public good similar to that of public health and see the viability to adopt mechanisms inspired by those used for public health.

\section{Reference}

AI100 S (Sep. 2016) One Hundred Year Study on Artificial Intelligence (AI100). Technical report, Stanford University.

Alkhatib H, Faraboschi P, Frachtenberg E, Kasahara H, Lange D, Laplante P, Merchant A, Milojicic D and Schwan K (Dec. 2014). IEEE CS 2022 Report. Technical report, IEEE Computer Society

Denning P J (2003) Great Principles of Computing. Communications of the ACM, 46 15-20

Harel D (2016) Niepce-Bell or Turing: How to Test Odor Reproduction? CoRR, abs/1603.08666. [LeCuri et al., 2015] LeCuri, Y., Bengio, Y., and Hiiiton, G. E. (2015). Deep Learning Nature 521 436-444. [Mandiant, 2014] Mandiant (2014). M-Trends Threat Report

Okhravi H, Rabe M A, Mayberry T J, Leonard W G, Hobson T R, Bigelow D and Streilein W W (Sep. 2013) Survey of Cyber Moving Target Techniques, http://www.dtic.mil/ cgi-bin/GetTRDoc ?Location $=\mathrm{U} 2 \&$ doc $=$ GetTRDoc . pdf\&AD=ADA591804

Preissl R, Worig T M, Datta P, Flickrier M, Singh R, Esser S K, Risk W P, Simon H D and Modha D S (2012) Compass: A Scalable Simulator for an Architecture for Cognitive Computing. In Proceedings of the International Conference on High Performance Computing, Networking, Storage and Analysis, SC '12, pages 54:1-54:11, Los Alamitos, CA, USA. IEEE Computer Society Press

Haddad R, Lapid H and Sobel N (2008) Measuring Smells. Current Opinion in Neurobiology 18 438-444
As mentioned before, the areas of computing or theory to practice is too vast to be covered in any one report. In the following chapters, the authors discuss areas like HPC and its cutting-edge applications, blockchain as a distributed trust management system, Big Data Analytics and its impact, ICT infrastructures and its applications, network computing (SDN) and its importance, future potential, etc. The issue also provides a glimpse into the challenges and suggestions (key takeaways) for innovative applications in science and society.

Sclmeider F and Savage S (Feb. 2009) Security is not a Commodity: The Road Forward for Cybersecurity Research, Computing Research Initiatives for the $21^{\text {st }}$ Century, http: //era.org/ccc/wp-content/uploads/sites/2/2015/05/ Cybersecurity.pdf

Schneider F B (2012) Blueprint for a science of Cybersecurity

Silver D, Huang A, Maddison C J, Guez A, Sifre L, van den Driessche G, Schrit-twieser J, Antonoglou I, Parmeershelvam V, Lanctot M, Dielemari S, Grewe D, Nham J, Kalch-brenner N, Sutskever I, Lillicrap T, Leach M, Kavukcuoglu K, Graepel T and Hassabis D (2016) Mastering the game of Go with deep neural networks arid tree search Nature 529 484-503

Tedre M (2014) The Science of Computing: Shaping a Discipline Chapman \& Hall/CRC

Tichenor S (June 7, 2007) Out-Compute to Out-Compete: Driving Competitiveness with Computational Modeling and Simulation

Turing AM (1937) On Computable Numbers, with an Application to the Eritscheidurigsprob-lem Proceedings of the London Mathematical Society s2-42 230-265

Turing A M (1950) Computing Machinery and Intelligence Mind LIX 433-460

Valiant L (2013) Probably Approximately Correct: Nature's Algorithms for Learning and Prospering in a Complex World. Basic Books, Inc., New York, USA. 